\title{
Establishing nursing adverse events' reporting content of hospital: using the Delphi method
}

Original article

Yue Liü, Hua-Ping Liub,*

${ }^{a}$ Rainbowfish Rehabilitation \& Nursing School, Hangzhou Vocational \& Technical College, Hangzhou, Zhejiang 310018, China

${ }^{b}$ School of Nursing, Peking Union Medical College, Beijing 100144, China

Received: 11 May 2020; Accepted: 11 June 2020; Published: 20 December 2020

\begin{abstract}
Objective: To develop nursing adverse events' reporting content of hospital.
Methods: The study included two phases. The first phase was to develop the category and definition of nursing adverse events that need to be reported through an expert meeting. The second phase was to develop every nursing adverse event's reporting content by using the Delphi method. In total, 8 experts attended the meeting and 15 experts conducted two rounds of consultation letter. Results: Nursing adverse events that need to be reported of hospital include pressure sore, fall/falling from bed, unplanned extubation, medication error, and accident. Reporting content of these events in detail had also been obtained, which was helpful for cause analysis systematically.

Conclusions: The reporting content of the nursing adverse event of hospital is established, and it is a basis for further study of the development of nursing adverse event reporting and feedback system.
\end{abstract}

Keywords: nursing adverse event $\bullet$ reporting content $\bullet$ expert meeting $\bullet$ Delphi method $\bullet$ Swiss Cheese Model $\bullet$ adverse event report

(c) Shanxi Medical Periodical Press.

\section{Introduction}

Medical adverse events would increase the clinical events, such as readmission, death, length of stay in hospital, and lead medical care providers to experience negative emotional symptoms..$^{1-4}$ Nursing adverse event would contribute to tell the risk, provide nurse training with reference, prevent the happening of similar events again, and offer evidence to continuing quality improvement. 5,6 There has not been unified or specific nursing adverse event reporting content in the Chinese mainland. Reporting content in that region generally had some shortcomings, for example, lacking unified or specific category of nursing adverse events and agreement on their definition, reporting content was not beneficial for data analysis or feedback, the discussion of systematic factors need to deepen. ${ }^{7,8}$ Swiss Cheese Model was a popular theoretical framework in the analysis of medical adverse events. ${ }^{9,10}$ According to this theory, adverse events were caused by the combined effect of several factors (systematic, personal, and

How to cite this article: Liu Y, Liu HP. Establishing nursing adverse events' reporting content of hospital: using the Delphi method. Front Nurs. 2020;4:337-344. 
environmental factors). These factors were like pieces of cheese that were stacked up. There were shortcomings in factors, like holes in cheese. When the holes in different pieces of cheese communicated accidentally and developed into a drill way, light would transmit through. This could be compared to the happening of adverse events. ${ }^{11}$ Based on the Swiss Cheese Model, this study developed a reason analysis part of reporting content from systematic, personal, and environmental factors, and aimed to establish nursing adverse events' reporting content of hospital.

\section{Methods}

\subsection{Expert meeting}

\subsubsection{Materials compilation for expert meeting}

Materials for the expert meeting were formed by literature review and reference of nursing adverse event reporting content of hospitals domestic and abroad.

\subsubsection{Selection of experts}

The inclusion criteria included experts who had worked in a hospital for more than 10 years, had a bachelor's or higher degree, were familiar with nursing quality control, and voluntary for the study. Totally eight experts attended the meeting. Seven experts were from a tertiary hospital, with one from the secondary hospital. Nursing quality control and improvement center approved of this selection of experts.

\subsection{Delphi method}

\subsubsection{Task team establishment}

The task team consisted of a graduate student, a tutor, and an instructor. Team members achieved missions including questionnaire design, expert selection, data collection, consultation information feedback, and statistical analysis.

\subsubsection{Questionnaire compilation for expert consultation}

The consultation questionnaire was developed based on literature review and reference of nursing adverse event reporting content at home and overseas. This questionnaire contained the following: (1) Instructions of the research background, time returned, contact information, and acknowledgment. (2)An evaluation form of nursing adverse event reporting content of hospital in detail, in which a score of 1-5 was assigned to denote the least to the most important item in sequence. The evaluated content was set by general reporting content and individual one. And individual reporting content was expanded from five events, namely pressure sore, fall/falling from bed, unplanned extubation, medication error, and accident. Meanwhile, every event's reporting content was comprised of the event description, reason analysis, and event consequence. According to the Swiss Cheese Model, the reason analysis portion covered systematic, personal, and environmental factors. (3) A form for gathering expert's general information, determining familiarity with consultation and self-assessment.

\subsubsection{Selection of experts}

The inclusion criteria included experts who had worked in hospital or nursing school for more than 10 years, had a bachelor's degree or higher, were familiar with nursing quality control, and voluntary for the study. The literature indicated that 15-50 experts would be appropriate. ${ }^{12}$ Totally 15 experts participated in the consultation. Among them, 12 were from tertiary hospitals, 2 from secondary hospital, and 1 from nursing school. Nursing quality control and improvement center approved this selection of experts.

\subsubsection{Two-round consultation}

In two rounds on consultation, questionnaires were distributed and retrieved by task team members in person.

\subsubsection{Establishment of filtering criteria}

Based on the literature review, items with a score of $<3.5$ or variable coefficient $>0.3$ would be removed. ${ }^{13}$ Items would be added, removed, or modified by opinions of the experts and discussion of task team members.

\subsection{Statistical analysis}

Data analysis was conducted by SPSS 19.0. Mean and standard deviation was used in descriptive analysis, which yielded general information, authority, and coefficient. Testing for coordination coefficient which was statistically different $(p<0.05)$ was considered significant.

\section{Results}

\subsection{General characteristics of the experts}

The mean age of experts who attended the meeting was $46.25 \pm 6.84$ years. Six experts had worked for $>20$ years and the mean of the whole working time was 
$26.12 \pm 7.40$ years. One expert had a doctor's degree, one had a master's degree, and the rest had a bachelor's degree. Among 8 experts who attended the meeting, the number of those who held senior, vice senior and the intermediate title was 4, 2 and 2, respectively.

The mean of Delphi consultation experts' age was $44.73 \pm 5.74$ years and the mean of their working time was $23.73 \pm 5.48$ years. One expert had a doctor's degree two had a master's degree, and the rest had a bachelor's degree. Among 15 Delphi consultation experts, the number of those who held senior, vice senior, and the intermediate title was 5,4 , and 6 , respectively.

\subsection{Initiative coefficients}

As all questionnaires were distributed and retrieved by task team members in person, $100 \%$ questionnaires had been collected in two-round consultation. A total of 14 experts $(93.33 \%)$ proposed constructive suggestions in the first round, with 12 experts $(80 \%)$ in the second round.

\subsection{Authority coefficients}

An authority coefficient $(\mathrm{Cr})$ depends on the familiarity with the field (Cs) and criterion (Ca), i.e., $\mathrm{Cr}=(\mathrm{Cs}+\mathrm{Ca}) / 2 .{ }^{12}$ Six degrees of familiarity were valued from 0.0 to 0.9 , indicating the lowest to the highest familiarity in an arithmetic sequence. ${ }^{12}$ Criteria were divided into more, medium, and less in sequence, including theoretical analysis $(0.3,0.2$, and 0.1$)$, practical experience $(0.5$, 0.4 , and 0.3$)$, literature at home and abroad $(0.1,0.1$, and 0.1$)$, and subjective judgment $(0.1,0.1$, and 0.1$)$. The authority coefficient of meeting experts was 0.869 , with 0.938 familiarity and 0.800 criterion. When it comes to Delphi consultation experts, the authority coefficient was 0.867 , with 0.900 familiarity and 0.833 criterion.

\subsection{Coordination coefficients}

Coordination depends on variable and coordination coefficients. ${ }^{12}$ The mean of variable coefficients in the first and second round was 0.21 and 0.14 , respectively. And the coordination coefficient in the first round was 0.543 , with which was 0.629 in the second round. Both coefficients were significantly different $(p<0.01)$. Tables 1 and 2 list the data obtained in detail.

\subsection{Category and definition of nursing adverse events that need reported}

Through the discussion of the expert meeting, nursing adverse events that need to be reported included pressure sore, fall/falling from bed, unplanned extubation,

\begin{tabular}{lccc}
\hline Round & Kendall's W & $\chi^{2}$ & $p$ \\
\hline 1 & 0.543 & 1377.607 & $0.00^{\star}$ \\
2 & 0.629 & 938.597 & $0.00 *$ \\
\hline
\end{tabular}

Note: ${ }^{\star} p<0.01$.

Table 1. Coefficients and significance testing results.

\begin{tabular}{|c|c|c|}
\hline Items & $\mathrm{M} \pm \mathrm{SD}$ & CV \\
\hline $\begin{array}{l}\text { General reporting content of nursing } \\
\text { adverse event }\end{array}$ & $4.28 \pm 0.76$ & 0.18 \\
\hline \multicolumn{3}{|c|}{ Individual reporting content of nursing adverse event } \\
\hline \multicolumn{3}{|l|}{ Pressure sore } \\
\hline Event description & $4.54 \pm 0.60$ & 0.14 \\
\hline Reason analysis & $4.50 \pm 0.60$ & 0.14 \\
\hline Event consequence & $4.24 \pm 0.75$ & 0.18 \\
\hline \multicolumn{3}{|l|}{ Fall/Falling from bed } \\
\hline Event description & $4.51 \pm 0.65$ & 0.15 \\
\hline Reason analysis & $4.49 \pm 0.62$ & 0.14 \\
\hline Event consequence & $4.38 \pm 0.69$ & 0.16 \\
\hline \multicolumn{3}{|l|}{ Unplanned extubation } \\
\hline Event description & $4.54 \pm 0.62$ & 0.14 \\
\hline Reason analysis & $4.53 \pm 0.60$ & 0.13 \\
\hline Event consequence & $4.46 \pm 0.69$ & 0.16 \\
\hline \multicolumn{3}{|l|}{ Medication error } \\
\hline Event description & $4.70 \pm 0.54$ & 0.12 \\
\hline Reason analysis & $4.46 \pm 0.66$ & 0.15 \\
\hline Event consequence & $4.57 \pm 0.57$ & 0.13 \\
\hline \multicolumn{3}{|l|}{ Accident } \\
\hline Event description and reason analysis & $4.59 \pm 0.56$ & 0.12 \\
\hline Event consequence & $4.49 \pm 0.63$ & 0.14 \\
\hline
\end{tabular}

Note: $\mathrm{CV}$ : coefficient of variation.

Table 2. Means, standard deviations, and variable coefficients of items.

medication error, and accident. The definitions of these events are shown in Table 3.

\subsection{Consultation}

According to the result of two-round consultation, the general reporting content of nursing adverse event contains the following: (1) Basic information of nursing adverse event, that is, time event happened, time event was found, time event was reported, patient's type, event finder, event reporter. (2) Nurse's basic information, that is, education background, professional title, personal working time, and working time in this department. (3) Patient's basic information, that is, sex, age, medical record No., nursing care level, education 


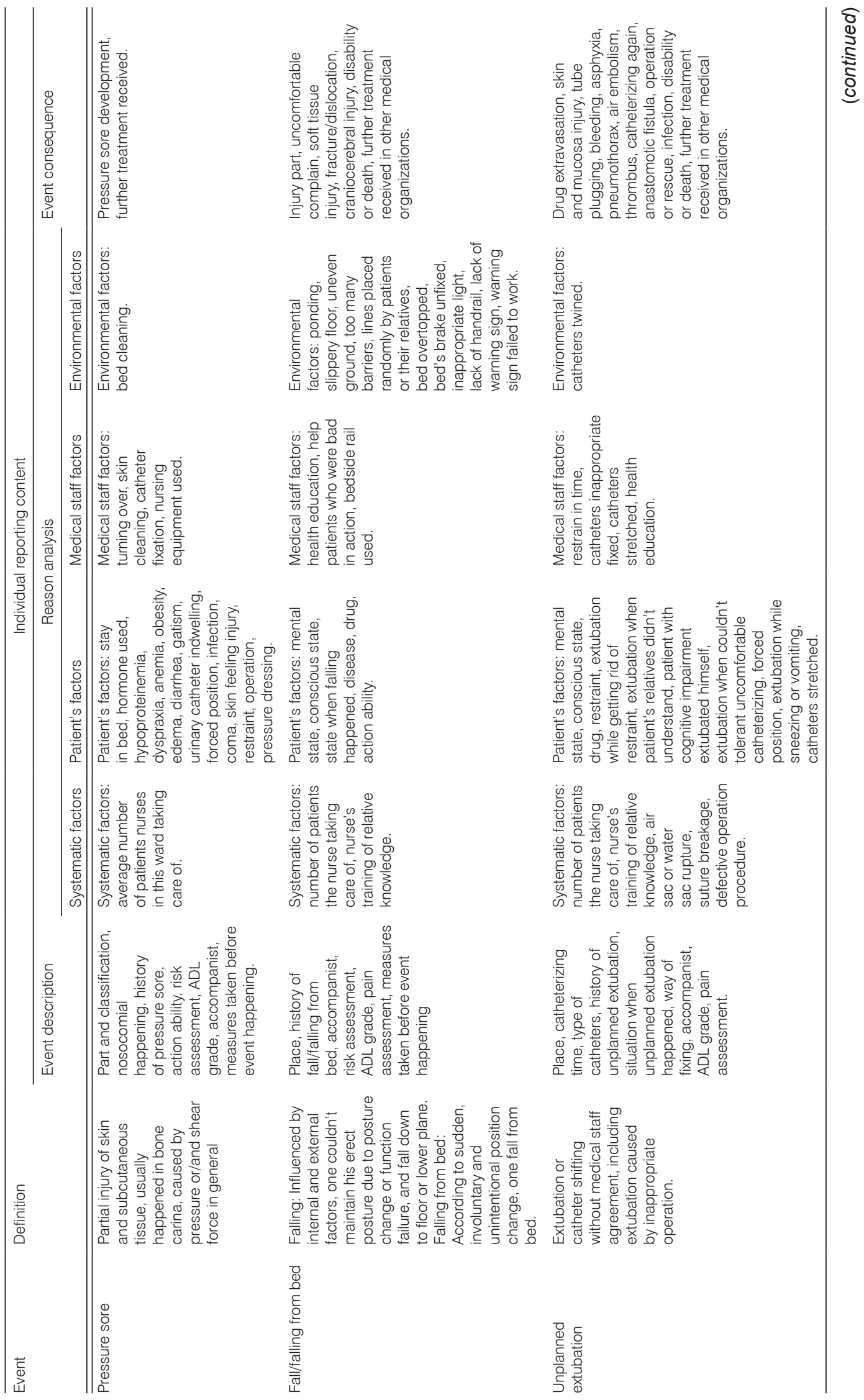




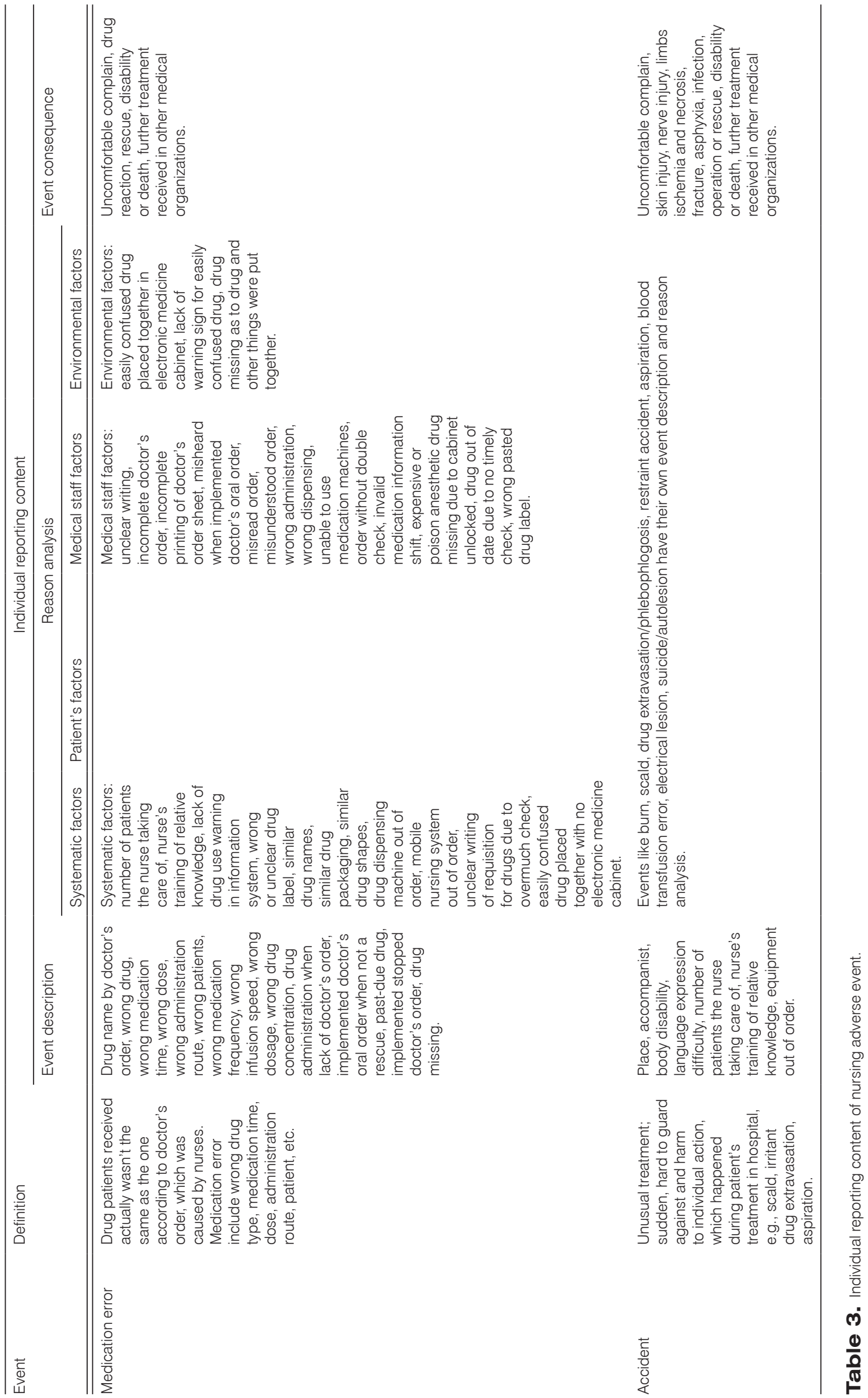


background, and chief medical diagnosis. (4) Brief description of the event. The individual reporting content of nursing adverse event is shown in Table 3.

\section{Discussion}

\subsection{Representation of experts}

The study invited experts who had worked for $>10$ years in clinical nursing, nursing administration, or nursing teaching research field. They were familiar with this study content and had gained in-depth knowledge of patient safety. All experts had a bachelor's or higher degree, intermediate or higher professional title, and $>10$-year working time. It could be seen that the representation of experts was fine.

\subsection{Reliability of results}

Items' adding or removing was according to scientific standard, and as the study was carried out on the basis of Swiss Cheese Model, it was reliable. First, initiative coefficients of experts were $100 \%$ in tworound consultation, which indicated that experts were interested and positive in this study. Second, the literature showed if authority coefficients were $>0.7$, the experts could be considered of high authority. ${ }^{13}$ Experts participated in this study all had authority coefficients which were $>0.7$. Third, it was shown that items retained should have a score $>3.5 .{ }^{14}$ There were six items which did not have scores demanded in the first round, and none in the second, illustrating the high concentration of experts' suggestion. Fourth, the coordination coefficient in the first and second round was 0.543 and 0.629 , respectively. Both coefficients were significantly different $(p<0.01)$. These coefficients demonstrated high coordination.

\subsection{General reporting content of nursing adverse event}

Compared with the content used now, time when event happened, was found and was reported was added. Literature indicated that the process of the adverse event "happen-be found-be reported" could demonstrate nurse's working attitude and busy level, normalization and complexity of reporting flow. These reflect systematic factors like human resource management, nurse training, and establishment of reporting flow as well. American Medical Management Committee emphasized that the key of medical adverse event is system. ${ }^{15}$ At the same time, attention from studies abroad had been paid to systematic factors in place of personal error ${ }^{16}$ and patient safety could be achieved by the improvement of system. ${ }^{17}$ This point of view was in accordance with the idea of developing reporting content in this study, that is, preventing the happening of the similar event again from telling and completing nursing systematic weak links.

\subsection{Individual reporting content of nursing adverse event}

\subsubsection{Individual reporting content of pressure sore}

The reason analysis was detailed in this study. First, objective indicators were added, for example, if the patient had hypoproteinemia, the nurse needs to write down the number of his total plasma protein. Second, the further cause was searched. For example, if the patient's bed wasn't clean, it could be in relation to "no timely delivery to ward of clean sheet", "the patient's noncooperation for sheet renewal", "negligence of nurses". These specific reasons would belong to systematic factors like the process of clothes and sheet cleaning, patient factors like their cooperation and medical staff factors like nurse's working attitude. It was indicated that the setting of objective indicators could help to reduce the influence of reporting content truth caused by subjective assumption. ${ }^{18}$ Further and deep reason analysis may lead to come up with the discussion of different factors based on the Swiss Cheese Model. As a result, adjustment and improvement of these factors is the next step.

\subsubsection{Individual reporting content of fall/falling from bed}

First, based on literature review, drug medication was described more in details comparing to reporting content domestic and abroad. ${ }^{19-22}$ Descriptions were added like drug's name, type, dose, frequency, time between drug administration and falling happened. Second, some reasons were explored further. For example, the situation of nurse's training was divided into three conditions, namely, participating in training, not participating in training and no training. If one joined training but lacks of relative knowledge, it could be in relation to systematic factors like the setting of training content and form, medical staff factors like nurse's halfheartedness of receiving training. If there was training while one did not go in for it, it could relate to systematic factors like unable to attend due to lack of human resources, medical staff factors like nurse's learning attitude. If there was no training of relative knowledge, it may be in connection with systematic factors like leaders' indifference to training. Along with the promotion of nonpunitive reporting culture in China, ${ }^{23}$ the significance of reporting adverse event is to prevent and control, 
especially lean attention on systematic safety. And this study did add analyzing personal error from the point of systematic factors.

\subsubsection{Individual reporting content of unplanned extubation}

Generally, reporting content abroad did not refer to the content of unplanned extubation, or sorted it into accident content. ${ }^{19-22}$ As a result, this event did not have its individual reporting content. In comparison with the content used now, specific situations of unplanned extubation were supplied, including air sac or water sac rupture, suture breakage, extubation while getting rid of restraint, extubation when patient's relatives did not understand, patient with cognitive impairment extubated himself, extubation when could not tolerant uncomfortable catheterization and catheters stretched. Literature indicated that there were various and complex situations of unplanned extubation. ${ }^{24,25}$ Meticulous situation reporting would be beneficial for analyzing from systematic factors like improving catheter quality, medical staff factors like offering patients and relatives health education and paying attention to patients with cognitive impairment.

\subsubsection{Individual reporting content of medication error}

Different from just describing medication error types in reporting content used now, this study listed reasons from systematic, personal, and environmental factors according to the Swiss Cheese Model. Some expressions were modified to correspond with Chinese mainland actual clinical situations. For example, "medication equipment" was altered as "injection pump and infusion pump"; "lack of electronic doctor's order screening and control system" was changed as "lack of drug use warning in information system". Establishment of reporting content should take nurses'

\section{References}

1. Lou FL, Tian H. Research progress on nursing adverse events. Chin Nurs Res. 2019;33:1726 1730 (in Chinese).

2. Sha HY, Yang $Y$, Wang $Y D, Y u$ YB, Zhang $X$. Research progress and prevention strategies on nursing adverse events. Chin Nurs Res. 2018;32:1531-1534 (in Chinese).

3. Lyu YL, Wang PH, Li SS, Li X. A qualitative study on nursing managers' attitudes towards the second comprehension into account. Too abstract content may be difficult for nurses to understand, which influences the reality and completeness of reporting content.

\subsubsection{Individual reporting content of accident}

As definitions domestic and abroad of accident were different, these regions' reporting contents were of little comparability. However, when it came to specific event reporting content, the result of this study was in accordance with that abroad. ${ }^{19-22}$ And reason analysis of events like burn, scald, drug extravasation/ phlebophlogosis, restraint accident, and aspiration were supplied based on the content used now.

\section{Conclusions}

Although the National Health Commission proposed to strengthen the reporting and management of medical adverse events, ${ }^{26}$ the specific reporting content of common nursing adverse events has not been clearly stipulated. In this study, especially reason analysis parts of reporting content were established based on the Swiss Cheese Model and nursing adverse events' reporting content of hospital was developed. This study made up the drawback of lacking normalization in the content now and would play a role in the development of nursing adverse event report and feedback system. The next step is to test and revise this reporting content in clinical practice.

\section{Ethical approval}

Ethical issues are not involved in this article.

\section{Conflicts of interest}

All contributing authors declare no conflicts of interest.

victim in adverse medical events. Chin $J$ Nurs. 2019;54:1210-1214 (in Chinese).

4. Ji DM, Luo CC, Li HF, Zhang L, Guo JC, Cai ZH. Application of special management in the control of adverse events in the care of elderly inpatients. $J$ Nurs Train. 2018,33:233-236 (in Chinese).

5. Zhang YY, Qin Y, Xie X. Study on the development of voluntary reporting system for nursing adverse events. J Nurs Train. 2017;32:222-225 (in Chinese). 
6. Zhang XJ, Zhang HZ, Zhang HJ, Li XW, Li NH, Wang $J Q$. Construction and discussion of management mode of adverse events of nursing based on systems theory. Chin Hosp Manage. 2017;37:6768 (in Chinese).

7. Thomas P. The Swiss cheese model of safety incidents: are there holes in the metaphor. BMC Health Serv Res. 2005;5:71.

8. Vincent C, Taylor-Adams S, Stanhope N. Framework for analysing risk and safety in clinical medicine. BMJ. 1998;316:1154-1157.

9. Chen MY, Xie M, Huang QS. Application of healthcare failure mode and effect analysis based on Swiss Cheese Model in the identification management. Mod Clin Nurs. 2019;18:58-65 (in Chinese).

10. Lu QH. Application of root cause analysis on the Swiss cheese model in the psychiatric nursing adverse events. Chin J Pract Nurs. 2015;31:26432646 (in Chinese).

11. Lu ZH, Wang Y, Guo XB, Yang XK. Research progress on the cause analysis models of nursing adverse events. J Nurs Sci. 2019;34:107-110 (in Chinese).

12. Li P. Statistical Application of Social Studies. Beijing: Social Science Academic Press; 2002:113-114 (in Chinese).

13. Reasons J. Human error: models and management. BMJ. 2002;320:768-770.

14. Institute of Medicine. Crossing the Quality Chasm: $A$ New Health System for the 21Century. Washington DC: National Academy Press; 2001.

15. Kohn LT, Corrigan JM, Donaldson MS. To Error is Human: Building A Safer Health System. Washington DC: National Academy Press; 2000:1-6.

16. Li CX, Xie CL, Fang W. The effect of nursing records form application in neurosurgery. Int $J$ Nurs. 2009;28:1460-1462 (in Chinese).
17. Cai J, Huang $\mathrm{CH}$. Design and clinical practice of the tabular nursing record form. Chin J Mod Nurs. 2010;16:2428-2430 (in Chinese).

18. Complaint Form. http://www.doh.wa.gov/portals/1/ Documents/Pubs/669277.pdf. Accessed April 7, 2019.

19. Patient Safety Incident Report Form. https://www. eforms.nrls.nhs.uk/staffreport/. Accessed April 7, 2019.

20. Clinical Incident Management System Data Request Process Patient Safety. http://www. safetyandquality.health.wa.gov.au/docs/aims/ CIMSDatarequestProcess.pdf. Accessed April 7, 2019.

21. Patient Safety Report form from Taiwan Patient Safety Report System. http://www.tpr.org.tw/dl/ TPR_5(13)C.pdf. Accessed May 26, 2019.

22. Guo XY, Ying QY, Kou DX. Application progress of zero defects theory in reducing nurses' medication errors. Chin J Mod Nurs. 2017;23:3313-3316 (in Chinese).

23. Cheng SZ, Huang TW, Cai JH, Tang CY, Hu LJ. Construction of non-punitive safety culture in nursing care. Chin Nurs Manage. 2018;18:13041307 (in Chinese).

24. Guo J. Cause of unplanned extubation of tracheal intubation patients in ICU and nursing safety management countermeasures. China Health Stand Manage. 2019;10:108-110 (in Chinese).

25. Yao W. Application of fishbone diagram analysis method in preventing unplanned extubation safety management. Nurs Pract Res. 2017;14:105-106 (in Chinese).

26. National Health Commission of the People's Republic of China. Measures for quality control of medical treatment. http://www.nhc.gov.cn/yzygj/s35 85/201610/5b61766ab433435fab6d5111c138d9f9. shtml. Accessed May 26, 2019. 